Department of Physics, G.B. Pant University of Agriculture \& Technology

(Pantnagar-263145, Uttarakhand, India; e-mail: bcchanyal@gmail.com, bcchanyal@gbpuat.ac.in)

\title{
A COMPLETE SET OF CONSERVATION LAWS PACS 02.10.De, 03.50.De OF DYONS WITH THE CLIFFORD-OCTONION ALGEBRA
}

\begin{abstract}
The Clifford-octonion algebra is the highest hypercomplex algebra over a real field with Clifford coefficient $(\varepsilon)$. The paper aims to apply the Clifford-octonion algebra (or simply called dualoctonion algebra) to describe the consistence theory of generalized electromagnetism and its conservation laws for dyons. The present paper describes the property of Lorentz invariance symmetries of dyons and their corresponding conservation laws. Moreover, we have studied the conservation of mass-energy, liner momentum, and angular momentum for four-dimensional particle dyons with the Clifford-octonion algebra. In generalized Clifford-octonion electromagnetic fields, we express the work-energy theorem and the linear momentum conservation theorem corresponding to the scalar and vector components of dyons, respectively. Finally, we have constructed the dual octonion form of the angular momentum conservation law, which represents an analog of the virial theorem in mechanics.
\end{abstract}

Ke ywords: dual octonion, dyons, angular momentum, conservation laws, virial theorem.

\section{Introduction}

In physics, the conservation laws are a fundamental frame to the understanding of the physical world, where we describe which physical processes can or cannot occur in the Nature. In general, the total quantity of the property governed by that law remains unchanged during physical processes. If we study the classical physics, the conservation laws include the conservation of mass (simply called matter), energy, linear momentum, angular momentum, and electric charge. Keeping in mind the sixteendimensional representation of the Clifford-octonion algebra and its space-time structure, the present paper describes the physical property of Lorentz invariance symmetries of dyons and their corresponding conservation laws. Moreover, we have studied the conservation of mass-energy, liner momentum, and angular momentum for four-dimensional particle dyons with the Clifford-octonion algebra. To describe every fundamental physical theory such as classical mechanics, quantum mechanics, theory of relativity, etc., we use a mathematical structure called Hilbert spaces (smooth manifolds) that are based on the real numbers, although the actual measurements of physical quantities are of finite accuracy and pre-

(C) B.C. CHANYAL, 2017

ISSN 2071-0194. Ukr. J. Phys. 2017. Vol. 62, No. 6 cision. In hypercomplex algebras over reals, we have only four sets of algebras, namely, the real algebra, complex algebra, algebra of quaternions $[1,2]$ and algebra of octonions [3, 4]. These four algebras are normed division algebras [5] and also alternative. The quaternion algebra is a four-dimensional algebra with associativity. The octonion division algebra is the highest normed division algebra. The octonionic algebra shows the eight-dimensional space-time structure and shares many attractive mathematical properties $[4,5]$. Octonions form a non-commutative nonassociative, but alternative algebra in mathematics. Moreover, Baez [6] discussed the octonion analysis and its multiplication properties. Recently, many authors [7-10] have already discussed the role of the eight-dimensional octonionic algebra in various branches of physics. Furthermore, a supersymmetric model and its various physical problems $[11,12]$ have been developed in terms of the octonion algebra. Let us introduce the octonion algebra, where the octonion variable $\xi \in \mathbb{O}$, is expressed as a set of eight-octons $\left(e_{0}\right.$ to $\left.e_{7}\right)$,

$\xi=\xi_{0} e_{0}+\sum_{j=1}^{7} \xi_{j} e_{j}$,

where $e_{j}(j=1,2,3, \ldots, 7)$ are imaginary octonion unities and $e_{0}$ is the real octonion unit element. The 
octonion unit element satisfies the following properties:

$$
\begin{aligned}
& e_{0}=1, \quad e_{j}^{2}=-1, \\
& e_{j} e_{k}=-\delta_{j k} e_{0}+f_{j k l} e_{l} \quad(j, k, l=1,2, \ldots, 7) \\
& {\left[e_{j}, e_{k}\right]=2 f_{j k l} e_{l}, \quad\left\{e_{j}, e_{k}\right\}=-\delta_{j k} e_{0},}
\end{aligned}
$$

where the structure constants $f_{j k l}$ are completely antisymmetric and take value 1 for $j k l=(123)$; (471); (257); (165); (624); (543); (736), and $\delta_{j k}$ is the usual Kronecker-Dirac delta-symbol.

\section{Clifford-Octonion Algebra}

The Clifford number [13] or simply called dual number defined by $Z^{D}$, associated with a two-dimensional commutative unital associative algebra over the real numbers. If we introduce the Clifford number on the octonion algebra, we obtain the Clifford-octonion algebra or dual-octonion algebra. However, the Clifford-octonion algebra is an extension of the octonion algebra, having a sixteen-dimensional structure with dual unity $\varepsilon$ that helps to study unified theories in physics. In order to study the dual-octonionic formalism for the electromagnetic field of dyons and keeping in mind the recent work on the dual octonion algebra [14-16], let us start with the following dual form of real numbers,

$Z^{D}=\left(Z+\varepsilon Z^{*}\right) \quad\left(\forall Z, Z^{*} \in \mathbb{R}\right)$,

and the Clifford unity $\varepsilon$ satisfies the following properties:

$\varepsilon^{2}=0, \quad \varepsilon \neq 0, \quad 0 \varepsilon=\varepsilon 0=0, \quad 1 \varepsilon=\varepsilon 1=\varepsilon$.

Moreover, the set of dual numbers forms a commutative ring having $\varepsilon Z^{*}$ as divisors of zero, i.e. not a field. Using Eq. (3), we may write the dual-octonionic function denoted by $W^{D} \sim W\left\{O_{j}, \varepsilon P_{j} ; \forall O_{j}, P_{j} \in \mathbb{O}\right.$ $\& j=0,1,2, \ldots, 7\}$ as

$W=\left(O_{0}+\varepsilon P_{0}\right) e_{0}+\sum_{j=1}^{7}\left(O_{j}+\varepsilon P_{j}\right) e_{j}=W_{0} e_{0}+W_{j} e_{j}$.

We can decompose both scalar and vector parts of the dual-octonion function, respectively, as

$W_{0} e_{0} \cong\left(S_{O}+\varepsilon S_{P}\right)=S^{D} \quad$ (Scalar part $)$,
$W_{j} e_{j} \cong\left(V_{O}+\varepsilon V_{P}\right)=V^{D} \quad$ (Vector part $)$.
Here, Eq. (6a) shows the real part of the dual-octonion function $W$ associated with a dual-scalar number, whereas the vector part given by $(6 \mathrm{~b})$ is associated with a dual vector. As such, the scalar multiplication operation with the dual-octonion function $W$ can be written as

$\lambda W=(\lambda O)+\varepsilon(\lambda P), \lambda \in \mathbb{R} ; O, P \in \mathbb{O}$.

Moreover, the octonion conjugate $\bar{W}$ and dual conjugate $W^{\varepsilon}$ of Eq. (5) may then be expressed as

$\bar{W}=\left(O_{0}+\varepsilon P_{0}\right) e_{0}-\sum_{j=1}^{7}\left(O_{j}+\varepsilon P_{j}\right) e_{j}=$
$=W_{0} e_{0}-W_{j} e_{j}$,
$W^{\varepsilon}=\left(O_{0}-\varepsilon P_{0}\right) e_{0}+\sum_{j=1}^{7}\left(O_{j}-\varepsilon P_{j}\right) e_{j}=$
$=W_{0}^{\varepsilon} e_{0}+W_{j}^{\varepsilon} e_{j}$,

where the dual property of the dual-octonionic function can be defined by

$\left\{\begin{array}{l}O_{0} e_{0}-\varepsilon P_{j} e_{j}=\left(\bar{W}+W^{\varepsilon}\right) / 2, \\ \varepsilon P_{0} e_{0}-O_{j} e_{j}=\left(\bar{W}-W^{\varepsilon}\right) / 2\end{array}\right.$

\section{Clifford-Octonionic Maxwell Equations}

Now, we can apply the dual-octonionic function $W$ to the electromagnetism of dyons. If we concern the classical electrodynamics of dyons (having both electric and magnetic charges) in the four-dimensional spacetime world, the differential operator $\square$, which has four-dimensional Euclidean space-time components, can be governed as

$\square=(\partial / \partial x) e_{1}+(\partial / \partial y) e_{2}+(\partial / \partial z) e_{3}-i(\partial / \partial t) e_{7}$,

and the dual-octonion potential of dyons [14] becomes

$V=\left[\left(\varphi_{m}+\varepsilon \varphi_{m}^{\prime}\right)+i e_{7}\left(\varphi_{e}+\varepsilon \varphi_{e}^{\prime}\right)\right] e_{0}+$

$+\sum_{j=1}^{3}\left[\left(A_{j}+\varepsilon A_{j}^{\prime}\right)+i e_{7}\left(B_{j}+\varepsilon B_{j}^{\prime}\right)\right] e_{j}$,

where $\left(\varphi_{e}, A_{j}\right)=\left(\varphi_{e}, \boldsymbol{A}\right)$ and $\left(\varphi_{m}, B_{j}\right)=\left(\varphi_{m}, \boldsymbol{B}\right)$ define, respectively, the octonion electric and magnetic potential components of dyons, while $\left(\varphi_{e^{\prime}}^{\prime} A_{j}^{\prime}\right)=$ $=\left(\varphi_{e^{\prime}}^{\prime}, \boldsymbol{A}^{\prime}\right)$ and $\left(\varphi_{m^{\prime}}^{\prime} B_{j}^{\prime}\right)=\left(\varphi_{m^{\prime}}^{\prime}, \boldsymbol{B}^{\prime}\right)$ indicate the Clifford components of the electric and magnetic potential of dyons. As such, the dual octonion form of 
a generalized electromagnetic field $(\Psi)$ can be expressed by the relation $(\vec{\cdot} V)=\Psi$. Here,

$\Psi=\sum_{j=1}^{3}\left(\mathbb{H}_{j}+i e_{7} \mathbb{E}_{j}\right) e_{j}=$

$=\sum_{j=1}^{3}\left[\left(H_{j}+\varepsilon H_{j}^{\prime}\right)+i e_{7}\left(E_{j}+\varepsilon E_{j}^{\prime}\right)\right] e_{j}$.

In relation (13), the components $\mathbb{E}_{j}:=\left(E_{j}+\varepsilon E_{j}^{\prime}\right)$ and $\mathbb{H}_{j}:=\left(H_{j}+\varepsilon H_{j}^{\prime}\right)$ define, respectively, the generalized dual-octonion components of the electric and magnetic fields of dyons. Here, the scalar components of the dual-octonionic generalized electromagnetic field vanish, if we apply the Lorentz gauge conditions, respectively, for the dynamics of the electric and magnetic charges of dyons. The dual-octonionic electric and magnetic field vectors can be expressed, respectively, as

$$
\left\{\begin{array}{l}
\left(E_{j}+\varepsilon E_{j}^{\prime}\right)=\left(-\boldsymbol{\nabla} \varphi_{e}-\frac{\partial \boldsymbol{A}}{\partial t}-(\boldsymbol{\nabla} \times \boldsymbol{B})\right)+ \\
+\varepsilon\left(-\boldsymbol{\nabla} \varphi_{e}^{\prime}-\frac{\partial \boldsymbol{A}^{\prime}}{\partial t}-\left(\boldsymbol{\nabla} \times \boldsymbol{B}^{\prime}\right)\right) \\
\left(H_{j}+\varepsilon H_{j}^{\prime}\right)=\left(-\boldsymbol{\nabla} \varphi_{m}-\frac{\partial \boldsymbol{B}}{\partial t}+(\boldsymbol{\nabla} \times \boldsymbol{A})\right)+ \\
+\varepsilon\left(-\boldsymbol{\nabla} \varphi_{m}^{\prime}-\frac{\partial \boldsymbol{B}^{\prime}}{\partial t}+\left(\boldsymbol{\nabla} \times \boldsymbol{A}^{\prime}\right)\right) .
\end{array}\right.
$$

From the definition of dual octonion current source given by the equation $(\square \Psi)=\Im$, where $\Im \in W$ is a dual octonion generalized current, we have

$$
\begin{aligned}
& \Im=\left[-\left(\rho_{m}+\varepsilon \rho_{m}^{\prime}\right)+i e_{7}\left(\rho_{e}+\varepsilon \rho_{e}^{\prime}\right)\right] e_{0}+ \\
& +\sum_{j=1}^{3}\left[\left(J_{j}+\varepsilon J_{j}^{\prime}\right)+i e_{7}\left(K_{j}+\varepsilon K_{j}^{\prime}\right)\right] e_{j},
\end{aligned}
$$

where the charge density and current density terms, respectively, $\left(\rho_{e}+\varepsilon \rho_{e}^{\prime}\right),\left(J_{j}+\varepsilon J_{j}^{\prime}\right)$, and $\left(\rho_{m}+\varepsilon \rho_{m}^{\prime}\right)$, $\left(K_{j}+\varepsilon K_{j}^{\prime}\right)$, are the dual-octonionic form of two fourcurrents associated with the electric charge and the magnetic charge (monopole) of dyons. Finally, we obtain the differential equations

$$
\begin{aligned}
& (\boldsymbol{\nabla} \cdot \boldsymbol{E})+\varepsilon\left(\boldsymbol{\nabla} \cdot \boldsymbol{E}^{\prime}\right)=\left(\rho_{e}+\varepsilon \rho_{e}^{\prime}\right), \\
& (\boldsymbol{\nabla} \cdot \boldsymbol{H})+\varepsilon\left(\boldsymbol{\nabla} \cdot \boldsymbol{H}^{\prime}\right)=\left(\rho_{m}+\varepsilon \rho_{m}^{\prime}\right), \\
& (\boldsymbol{\nabla} \times \boldsymbol{E})+\boldsymbol{\varepsilon}\left(\boldsymbol{\nabla} \times \boldsymbol{E}^{\prime}\right)= \\
& =-\left(\frac{\partial \boldsymbol{H}}{\partial t}+\varepsilon \frac{\partial \boldsymbol{H}^{\prime}}{\partial t}\right)-\left(\boldsymbol{K}+\varepsilon \boldsymbol{K}^{\prime}\right),
\end{aligned}
$$

$(\boldsymbol{\nabla} \times \boldsymbol{H})+\varepsilon\left(\nabla \times \boldsymbol{H}^{\prime}\right)=$

$=\left(\frac{\partial \boldsymbol{E}}{\partial t}+\varepsilon \frac{\partial \boldsymbol{E}^{\prime}}{\partial t}\right)+\left(\boldsymbol{J}+\varepsilon \boldsymbol{J}^{\prime}\right)$,

which presents the well-known generalized DiracMaxwell's equations in terms of the dual octonion algebra [16]. Therefore, the dual-octonionic Hilbert space formulation is compact and consistent.

\section{Energy and Linear \\ Momentum Conservation Laws}

In this section, we represent the role of the dual-octonion algebra in symmetry and conservation laws. Since the forms of the laws of conservation of energy and momentum are important for the electromagnetic field [16], let us start with the dual-octonionic field equation $\Psi(\bullet \Psi)=\Psi \Im$, where the left-hand term is

$$
\begin{aligned}
& \Psi(\bullet \Psi)=S^{D}(\alpha, \beta)+V^{D}(\boldsymbol{\Gamma}, \boldsymbol{\Lambda}) \equiv \\
& \equiv\left(\alpha e_{0}+\beta e_{7}\right)+\sum_{j=1}^{3}\left(\boldsymbol{\Gamma} e_{j}+\boldsymbol{\Lambda} e_{j+3}\right),
\end{aligned}
$$

where $S^{D}(\alpha, \beta)$ denotes a dual scalar function, while $V^{D}(\boldsymbol{\Gamma}, \boldsymbol{\Lambda})$ denotes a dual vector function in the generalized dual-octonion field. Thus, the components are

$$
\begin{aligned}
& \alpha=\mathbb{H} \cdot \frac{\partial \mathbb{H}}{\partial t}+\mathbb{E} \cdot \frac{\partial \mathbb{E}}{\partial t}+\mathbb{H} \cdot(\nabla \times \mathbb{E})-\mathbb{E} \cdot(\nabla \times \mathbb{H}), \\
& \beta=\mathbb{H} \cdot \frac{\partial \mathbb{E}}{\partial t}+\mathbb{E} \cdot \frac{\partial \mathbb{H}}{\partial t}-\mathbb{H} \cdot(\boldsymbol{\nabla} \times \mathbb{H})+\mathbb{E} \cdot(\boldsymbol{\nabla} \times \mathbb{E}), \\
& \Gamma=\mathbb{H} \times \frac{\partial \mathbb{E}}{\partial t}-\mathbb{E} \times \frac{\partial \mathbb{H}}{\partial t}-\mathbb{H} \times(\boldsymbol{\nabla} \times \mathbb{H})- \\
& -\mathbb{E} \times(\boldsymbol{\nabla} \times \mathbb{E})+\mathbb{H}(\boldsymbol{\nabla} \cdot \mathbb{H})+\mathbb{E}(\boldsymbol{\nabla} \cdot \mathbb{E}),
\end{aligned}
$$

$\Lambda=\mathbb{E} \times \frac{\partial \mathbb{E}}{\partial t}-\mathbb{H} \times \frac{\partial \mathbb{H}}{\partial t}-\mathbb{H} \times(\nabla \times \mathbb{E})-$

$-\mathbb{E} \times(\boldsymbol{\nabla} \times \mathbb{H})-\mathbb{H}(\boldsymbol{\nabla} \cdot \mathbb{E})+\mathbb{E}(\boldsymbol{\nabla} \cdot \mathbb{H})$.

Similarly, the right-hand side of the dual-octonionic field equation, $\Psi \Im$, may be expressed in terms of the dual-scalar and dual-vector parts, i.e.

$$
\begin{aligned}
& \Psi \Im=S^{D}\left(\alpha^{\prime}, \beta^{\prime}\right)+V^{D}\left(\boldsymbol{\Gamma}^{\prime}, \boldsymbol{\Lambda}^{\prime}\right) \equiv \\
& \equiv\left(\alpha^{\prime} e_{0}+\beta^{\prime} e_{7}\right)+\sum_{j=1}^{3}\left(\boldsymbol{\Gamma} e_{j}+\boldsymbol{\Lambda}^{\prime} e_{j+3}\right),
\end{aligned}
$$


where the components become

$\alpha^{\prime}=\mathbb{H} \cdot \mathbb{K}+\mathbb{E} \cdot \mathbb{J}$,

$\beta^{\prime}=\mathbb{H} \cdot \mathbb{J}+\mathbb{E} \cdot \mathbb{K}$,

$\Gamma^{\prime}=\rho_{m} \mathbb{H}+\rho_{e} \mathbb{E}-\mathbb{H} \times \mathbb{J}-\mathbb{E} \times \mathbb{K}$,

$\Lambda^{\prime}=\rho_{e} \mathbb{H}-\rho_{m} \mathbb{E}+\mathbb{H} \times \mathbb{K}-\mathbb{E} \times \mathbb{J}$.

Since the energy is a scalar quantity, we equate, in this case, the scalar components of the dual-octonion coefficients $\alpha=\alpha^{\prime}$ and obtain the total rate of doing work by the fields in a finite volume $d \tau$,

$\int\left[\frac{1}{2} \frac{\partial \mathbb{E}^{2}}{\partial t}+\frac{1}{2} \frac{\partial \mathbb{H}^{2}}{\partial t}+\nabla \cdot(\mathbb{E} \times \mathbb{H})\right] d \tau=$

$=\int(\mathbb{H} \cdot \mathbb{K}+\mathbb{E} \cdot \mathbb{J}) d \tau$.

The above equation (21) shows the work-energy theorem simply called the Poynting theorem for the generalized dual-octonion electrodynamics of dyons. As such, the dual-octonionic energies due to electric and magnetic fields are defined, respectively, as

$\Omega_{e}=\frac{1}{2} \int \mathbb{E}^{2} d \tau, \quad \Omega_{m}=\frac{1}{2} \int \mathbb{H}^{2} d \tau$.

Accordingly, the total energy density in the generalized dual-octonion electromagnetic fields of dyons can be represented as

$\Omega_{e m}=\frac{1}{2} \int\left(\mathbb{E}^{2}+\mathbb{H}^{2}\right) d \tau$.

In Eq. (21), the term $(\mathbb{E} \times \mathbb{H}) \rightarrow \mathbb{S}$ shows the dual-octonionic Poynting vector, which represents the directional energy flux density of the generalized electromagnetic field of dyons. Therefore, the conservation of energy is governed by the following equation:

$\frac{d \Omega}{\partial t}=\frac{\partial \Omega_{e m}}{\partial t}+\nabla \cdot \mathbb{S}-(\mathbb{H} \cdot \mathbb{K}+\mathbb{E} \cdot \mathbb{J})$.

On the other hand, we know that the momentum is a vector quantity so that, in view of the conservation of momentum for the generalized electrodynamics of dyons, we may equate the vector components of the dual-octonionic coefficients, $\boldsymbol{\Gamma}=\boldsymbol{\Gamma}^{\prime}$, and get the following equation:

$$
\begin{aligned}
& -\frac{\partial \mathbb{S}}{\partial t}+\frac{1}{2} \boldsymbol{\nabla}\left(\mathbb{E}^{2}+\mathbb{H}^{2}\right)-(\mathbb{H} \cdot \boldsymbol{\nabla}) \mathbb{H}-(\mathbb{E} \cdot \boldsymbol{\nabla}) \mathbb{E}- \\
& -\mathbb{H}(\boldsymbol{\nabla} \cdot \mathbb{H})-\mathbb{E}(\boldsymbol{\nabla} \cdot \mathbb{E})= \\
& =\rho_{m} \mathbb{H}+\rho_{e} \mathbb{E}-\mathbb{H} \times \mathbb{J}-\mathbb{E} \times \mathbb{K} .
\end{aligned}
$$

The divergence of the Maxwell stress tensor $(\boldsymbol{\nabla} \cdot \overleftrightarrow{\boldsymbol{T}})$ is associated with its $j$ th component as

$$
\begin{aligned}
& \boldsymbol{\nabla} \cdot \stackrel{\leftrightarrow}{\boldsymbol{T}}=\left[(\boldsymbol{\nabla} \cdot \mathbb{E}) \mathbb{E}_{j}+(\mathbb{E} \cdot \boldsymbol{\nabla}) \mathbb{E}_{j}-\frac{1}{2} \boldsymbol{\nabla}_{j}\left(\mathbb{E}^{2}\right)\right]+ \\
& +\left[(\boldsymbol{\nabla} \cdot \mathbb{H}) \mathbb{H}_{j}+(\mathbb{H} \cdot \boldsymbol{\nabla}) \mathbb{H}_{j}-\frac{1}{2} \boldsymbol{\nabla}_{j}\left(\mathbb{H}^{2}\right)\right]
\end{aligned}
$$

and the generalized Lorentz force density for dyons, suggested by this symmetry, is expressed as

$\boldsymbol{f}^{e m}=\left(\rho_{e} \mathbb{E}+\mathbb{J} \times \mathbb{H}\right)+\left(\rho_{m} \mathbb{H}-\mathbb{K} \times \mathbb{E}\right)$.

Finally, from Eq. (25), we obtain the following compact relation, which leads to the conservation of momentum for dyons in case of the dual-octonion structure:

$\nabla \stackrel{\leftrightarrow}{\boldsymbol{T}}+\frac{\partial \mathbb{S}}{\partial t}+\boldsymbol{f}^{e m}=0$

Furthermore, if no external forces are applied, i.e. $\boldsymbol{f}^{e m}=0$, we obtain the local statement of the conservation of momentum of the electromagnetic field of dyons and the continuity equation. On the other hand, if we compare the remaining dual-octonion coefficients $\beta=\beta^{\prime}$, and $\Lambda=\Lambda^{\prime}$, we find a complete set of generalized Dirac-Maxwell equations of dyons.

\section{Angular Momentum Conservation Law}

In order to express the dual-octonionic conservation law of angular momentum for dyons, let us start with the components of the stress tensor,

$\boldsymbol{T}_{k l}=\delta_{k l} \Omega_{e m}-\mathbb{E}_{k} \mathbb{E}_{l}+\mathbb{H}_{k} \mathbb{H}_{l}$.

Here, we have to note that the stress tensor is symmetric, i.e. $\boldsymbol{T}_{k l}=\boldsymbol{T}_{l k}$, which is required in order to obtain a local conservation law for angular momentum. As such, the trace of the stress tensor $\overleftrightarrow{\boldsymbol{T}}$ (the sum of the diagonal elements $\boldsymbol{T}_{k k}$ ) is simply defined in terms of the energy density $\Omega_{e m}$ as

$\operatorname{Tr}(\overleftrightarrow{\boldsymbol{T}})=\sum_{k} \boldsymbol{T}_{k k}=\Omega_{e m}$

Moreover, in the tensor form, the conservation relation (28) becomes

$\nabla_{l} \boldsymbol{T}_{l k}+\frac{\partial \mathbb{S}_{k}}{\partial t}+\boldsymbol{f}_{k}^{e m}=0$.

ISSN 2071-0194. Ukr. J. Phys. 2017. Vol. 62, No. 6 
The torque $\tau$ for dyons in terms of the dual-octonionic form can be written as

$\tau=\boldsymbol{r} \times \boldsymbol{F}=\int(\boldsymbol{d} \boldsymbol{r}) \boldsymbol{r} \times \boldsymbol{f}^{e m}$.

Correspondingly, the torque density (force density moment) can be written in the component form as

$\left(\boldsymbol{r} \times \boldsymbol{f}^{e m}\right)_{i}=\boldsymbol{\epsilon}_{i j k} \boldsymbol{x}_{j} \boldsymbol{f}_{k}^{e m}$,

where $\boldsymbol{\epsilon}_{i j k}$ denotes the Levi-Civita tensor, which is totally antisymmetric, i.e.

$\boldsymbol{\epsilon}_{i j k}=-\boldsymbol{\epsilon}_{j i k}=-\boldsymbol{\epsilon}_{k j i}=-\boldsymbol{\epsilon}_{i k j}=\boldsymbol{\epsilon}_{j k i}$,

is normalized by $\boldsymbol{\epsilon}_{123}=1$, and vanishes if any two indices are equal. Thus, the dual-octonionic torque density for dyons can be obtained by taking the moment of the force density,

$\boldsymbol{\nabla}_{j}\left(\boldsymbol{x}_{j} \boldsymbol{T}_{l k}\right)+\frac{\partial}{\partial t}\left(\boldsymbol{x}_{j} \mathbb{S}_{k}\right)-\boldsymbol{T}_{j k}+\boldsymbol{x}_{j} \boldsymbol{f}_{k}^{e m}=0$,

where we have used that $\nabla_{l} \boldsymbol{x}_{j}=\boldsymbol{\delta}_{l j}$. Finally, we get

$\boldsymbol{\nabla}_{l}\left(\boldsymbol{\epsilon}_{i j k} \boldsymbol{x}_{j} \boldsymbol{T}_{l k}\right)+\frac{\partial}{\partial t}\left(\boldsymbol{\epsilon}_{i j k} \boldsymbol{x}_{j} \mathbb{S}_{k}\right)+\boldsymbol{\epsilon}_{i j k} \boldsymbol{x}_{j} \boldsymbol{f}_{k}^{e m}=0$.

We note that $\boldsymbol{T}_{k l}$ is symmetric. Thus, this symmetry is required for the existence of a local conservation law of angular momentum. Therefore, we identify the following electromagnetic angular momentum quantities in the dual-octonionic form:

$\Im=r \times \mathbb{S}$

( $\rightarrow$ dual octonionic angular momentum density),

$\Re_{i j}=\epsilon_{j k l} \boldsymbol{x}_{k} \boldsymbol{T}_{i l}$,

( $\rightarrow$ dual octonionic angular momentum flux density)

Furthermore, if $j=k$, then we obtain the following important equation:

$\boldsymbol{\nabla} \cdot(\overleftrightarrow{\boldsymbol{T}} \cdot \boldsymbol{r})-\frac{\partial}{\partial t}(\boldsymbol{r} \cdot \mathbb{S})-\Omega_{e m}+\boldsymbol{r} \cdot \boldsymbol{f}^{e m}=0$

Equation (38) represents the electromagnetic virial theorem in the dual-octonionic formulation, which is an analog to the mechanical virial theorem of Rudolf Clausius [17]. The advantage of the Clifford-octonion algebra is that it also can be used in quantum computation systems (quantum computers) that make direct use of quantum-mechanical phenomena [18], such as superposition and entanglement, to perform the operations on data. On the other hand, in view of the 16-dimensional representation, the sedenion [19] is another powerful alternative hypercomplex algebra satisfying the non-associative and non-commutative property over reals. We also can use the sedenion algebra for the unification of fundamental forces in a single framework.

\section{Conclusion}

In mathematical physics, the Clifford-octonion algebra is a generalized version of octonion algebra, which exhibits the 16-dimensional structure of Euclidean space-times in the universe. This 16-dimension algebra represents some classical field equations of physical variables. Here, we have assumed that the dual-octonion (higher dimensional structure) representation presents a possibility to show the existence of a magnetic monopole along with the electric charge. We have studied the generalized EMfield equations and the current source equations of dyons in terms of dual-octonions. Therefore, we have investigated the compact form of symmetric generalized Dirac-Maxwell's equations of dyons with the dual-octonion algebra. The dual-octonion form of the work-energy theorem or Poynting theorem of dyons has been developed. The beauty of the dual octonion representations is that the scalar coefficient represents the Poynting theorem, and the vector coefficient shows the conservation of liner momentum. In view of the rotational symmetry, we have discussed the angular momentum conservation laws for dyons in a compact way with the dual-octonion algebra. We also have given the electromagnetic virial theorem in the dual-octonionic formulation, which is analogous to the mechanical virial theorem. The important conclusion of the dual-octonion formulation is the existence of a monopole and dyons in a higher dimensional Grand Unified Theory (GUT). Like the octonion formalism [20,21], the presented 16-dimensional theoretical model can also be used in various branches of physics, e.g., elementary particle physics, nuclear physics, gravitational physics, etc.

1. W.R. Hamilton. Elements of Quaternions (Chelsea, 1969).

2. P.G. Tait. An Elementary Treatise on Quaternions (Oxford Univ. Press, 1875). 
3. R.P. Graves. Life of Sir William Rowan Hamilton (Arno Press, 1975).

4. J. Schray. Octonions and Supersymmetry. Ph. D. Thesis (Oregon State Univ., 1994).

5. A. Cayley. An Jacobi's elliptic functions, in reply to the Rev. B. Bornwin; and on quaternion. Phil. Mag. 26, 208 (1845).

6. J.C. Baez. The octonions. Bull. Amer. Math. Soc. 39, 145 (2001).

7. M. Tanışlı, M.E. Kansu. Octonionic Maxwell's equations for bi-isotropic media. J. Math. Phys. 52, 053511 (2011).

8. M. Tanışlı, M.E. Kansu, S. Demir. Reformulation of electromagnetic and gravito-electromagnetic equations for Lorentz system with octonion algebra. Gen. Relativ. Gravit. 46, (2014).

9. B.C. Chanyal, P.S. Bisht, O.P.S. Negi. Generalized octonion electrodynamics. Int. J. Theor. Phys. 49, 1333 (2010).

10. B.C. Chanyal, P.S. Bisht, Li Tianjun, O.P S. Negi. Octonion quantum chromodynamics. Int. J. Theor. Phys. 51, 3410 (2012).

11. J. Lukierski, F. Toppan, Generalized space-time supersymmetries, division algebras and octonionic M-theory. Phys. Lett. B 539, 266 (2002).

12. R. Foot, G.C. Joshi. Space-time symmetries of superstring and Jordan algebras. Int. J. Theor. Phys. 28, 1449 (1989).

13. W. Clifford. Preliminary sketch of biquaternions. Proc. London Math. Soc. 4, 381 (1873).

14. B.C. Chanyal, S.K. Chanyal, Ö. Bektaş, S. Yüce. A new approach on electromagnetism with dual number coefficient octonion algebra. Int. J. Geom. Meth. Mod. Phys. 13, 1630013 (2016).

15. B.C. Chanyal. Dual octonion electrodynamics with the massive field of dyons. J. Math. Phys. 57, 033503 (2016).

16. B.C. Chanyal, S.K. Chanyal. Dual number coefficient octonion algebra, field equations and conservation laws.
Analysis Math. Phys. (2016), (Online Published) DOI: 10.1007/s13324-016-0144-6.

17. R. Clausius. On a mechanical theorem applicable to heat Phil. Magaz. 40, 122 (1870)

18. B.C. Chanyal. Octonion generalization of Pauli and Dirac matrices. Int. J. Geom. Meth. Mod. Phys. 12, 1550007 (2015).

19. B.C. Chanyal. Sedenion unified theory of gravi-electromagnetism. Indian J. Phys. 88, 1197 (2014).

20. B.C. Chanyal, V.K. Sharma, O.P.S. Negi. Octonionic gravi-electromagnetism and dark matter. Int. J. Theor. Phys. 54, 3516 (2015).

21. B.C. Chanyal. Generalized Klein-Gordon field equations with octonion space-time (OST) algebra. Chinese J. Phys. 55, 432 (2017).

Received 08.03.17

\section{Б.С. Чанял}

\section{ПОВНИЙ НАБІР ЗАКОНІВ ЗБЕРЕЖЕННЯ}

\section{ДЛЯ ДІОНІВ В АЛГЕБРІ КЛІФФОРДА ОКТОНІОНІВ}

$\mathrm{P}$ е $з$ ю м е

Алгебра Кліффорда октоніонів (або просто дуальна алгебра октоніонів) - це вища гіперкомплексна алгебра над дійсним полем з коефіцієнтом Кліффорда $(\varepsilon)$. Алгебру застосовано для опису узгодженої теорії узагальненого електромагнетизму і його законів збереження для діонів. Описано властивість симетрій Лоренц-інваріантності і відповідні закони збереження для діонів. Вивчено збереження масиенергії, лінійного імпульсу і кутового моменту для чотиривимірних діонів. Для узагальнених електромагнітних полів доведено теорему роботи-енергії і теорему лінійного імпульсу, що відповідають скалярній і векторній компонентам діонів, відповідно. Побудовано дуальну октоніонну форму закона збереження для лінійного імпульсу, що являє аналог теореми виріала в механіці. 